\title{
Unusual combination of muscular and arterial variations in the upper extremity: a case report of a variant palmaris longus and an additional tendinous portion of the flexor carpi ulnaris together with a persistent median artery
}

\author{
Georgi P. Georgiev, Lazar Jelev, Wladimir A. Ovtscharoff \\ Department of Anatomy, Histology and Embryology, Medical University of Sofia, Sofia, Bulgaria
}

\begin{abstract}
In this report we describe an unusual combination of anatomical variations in the left upper extremity. A rare case of a variant palmaris longus muscle, an unknown variation of the flexor carpi ulnaris muscle and a persistent median artery were discovered during routine anatomical dissection. The anatomical aspects of these variations have been discussed in detail. We also review the clinical literature and presented their possible clinical implications.
\end{abstract}

Key words: multiple anatomic variations; palmaris longus; flexor carpi ulnaris; persistent median artery; clinical significance Anatomy 2009; 3: 58-61, (C) 2009 TSACA

\section{Introduction}

Variations in the arteries, nerves and muscles of the upper limb have both academic and clinical relevance. ${ }^{1}$ Different variations of the palmaris longus muscle, the flexor carpi ulnaris muscle and the persistent median artery have been well documented in the anatomical literature..$^{1-7}$ These variations could be of importance for the clinical practice and there are many clinical case reports presenting their significance for orthopedics, plastic surgeons and neurologists. ${ }^{8-14}$ Therefore, knowledge of these anomalous structures and especially cases with multiple coexisting variations, although rare, is important not only to anatomists but also to clinicians in their routine practice.

In this report, we describe the co-existence of the aforementioned variations and emphasize on their clinical implications.

\section{Case Report}

Multiple anatomical variations in the forearm were discovered on the left side of a 70-year-old male cadaver during routine dissection. No variations in the contralateral right upper extremity were discovered. The left-side variations were as follows.

\section{Variation of the palmaris longus}

The variant palmaris longus arose as usual from the medial epicondyle of the humerus, with the other flexor muscles of the forearm and from the antebrachial fascia. Its proximal muscle belly prolonged downwards in two separate well-formed flattened tendons - lateral and medial. The lateral tendon passed superficial to the flexor retinaculum and inserted into the palmar aponeurosis; 
the medial one distally reached the anterior aspect of the proximal part of the flexor retinaculum (Figure 1A).

\section{Variation of the flexor carpi ulnaris}

In our case, an additional tendinous portion of the flexor carpi ulnaris was detected. There was an usually structured flexor carpi ulnaris taking origin proximally with two heads - humeral and ulnar. They formed a muscular belly ending in a strong tendon that inserted into the pisiform bone. Additionally, beneath the muscular belly and the tendon of the flexor carpi ulnaris an aberrant well-separate flat tendon was detected. It arose from the medial epicondyle of the humerus together with the flexor carpi ulnaris, passed downwards in close relation to its posterior surface and attached to the pisiform bone. In the middle of the forearm, a small muscular slip from the flexor carpi ulnaris joined the aberrant tendon (Figure 1B).

\section{Median artery}

The median artery arose from the ulnar artery at the same level as the common interosseous artery. It descended to the wrist on the superficial aspect of the flexor pollicis longus and deep to the flexor digitorum superficialis. At the wrist the artery passed deep to the flexor retinaculum, running to the carpal tunnel to enter the palmar aspect of the hand and prolonged as the princeps pollicis artery. Its branches were distributed to the skin and subcutaneous tissue of the thumb and the index finger (Figure 1C).

\section{Discussion}

We found an unusual combination of unilateral muscular and arterial variations in the distal part of the forearm - duplication of the distal palmaris longus tendon, variation of the flexor carpi ulnaris and a persistent median artery. In the literature there are some reports of an aberrant palmaris longus coexisting with persistent median artery. ${ }^{15,16}$ However, such combination of a duplication of the palmaris longus tendon, a persistent median artery and a variant flexor carpi ulnaris, as described by ours, is reported for the first time. We review the anatomical description of the variant findings and emphasize on their potential clinical significance.

The palmaris longus is the most variable muscle in the human arm and one of the most variable muscles in the human body. ${ }^{7,13,14,17}$ The most common variations are its absence (around 10\%), digastric muscle, reversed muscle, bifid reversed variation and duplication, as in our
Figure 1. Photograph of the variant findings. A: palmaris longus muscle (PL) with its medial tendon (MT) and lateral tendon (LT). B: flexor carpi ulnaris (FCU) and its small muscular slip (arrowhead) joining the aberrant tendon (arrows). C: Median artery (MA) passing with the median nerve (MN).

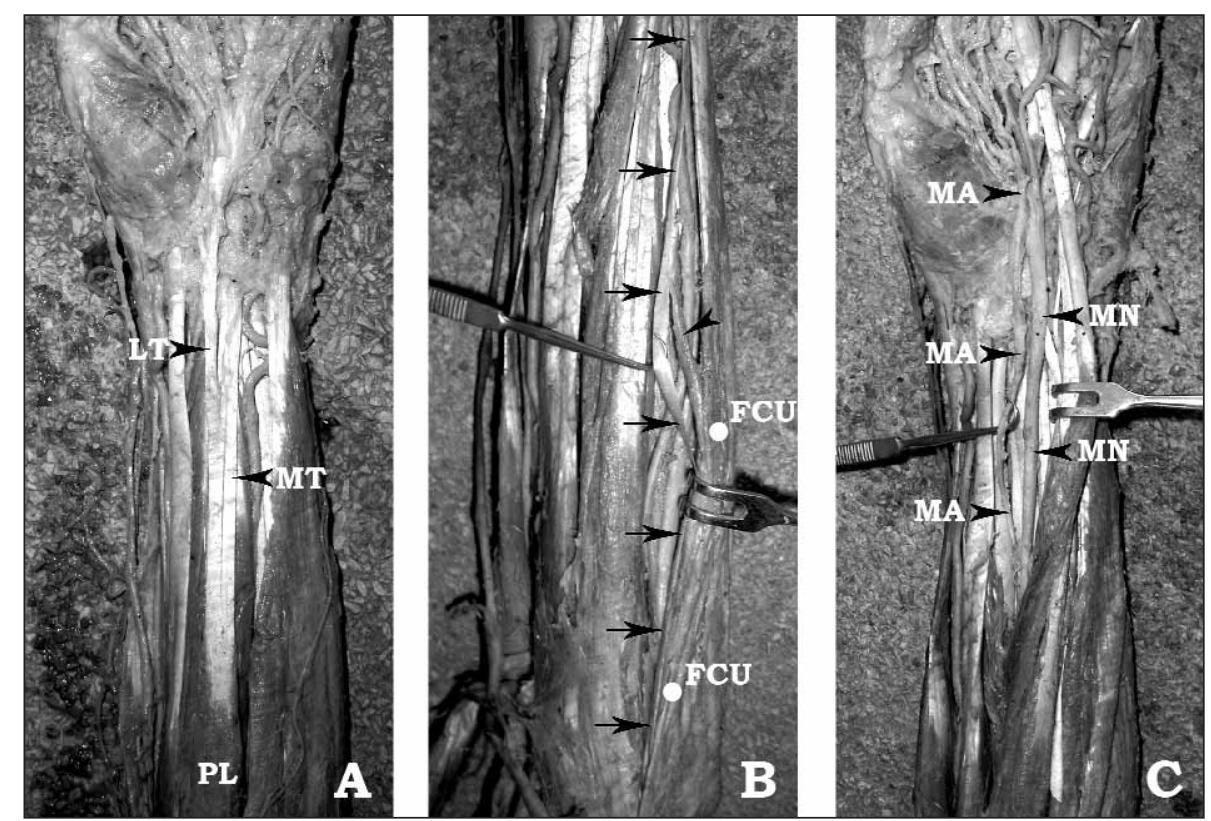


case. $^{7,13,14,17-21}$ According to Paraskevas et al., the duplication of palmaris longus vary between $0.8-3.1 \%{ }^{22}$ Numerous reports of the variations of this muscle and their clinical significance could be found in the literature. $^{7,13,14,17,18}$ There are many reports of median and /or ulnar nerve compression due to the existence of a variant palmaris longus. ${ }^{7,13,14,17,18}$ In clinical practice, the aberrant palmaris longus could also be incidentally found during clinical examination without provoking clinical symptoms and may simulate a soft tissue tumor. ${ }^{18}$ The palmaris longus is often considered as an ideal donor in reconstructive and plastic surgery and knowledge of its variations is important to provide safe and successful surgical procedures. ${ }^{13}$ This muscle could be used for tendon grafts in replacement of the long flexors of the fingers, the flexor pollicis longus tendon, dorsal finger injuries involved with both soft tissue loss and extensor tendon defects. ${ }^{7}$ It is also utilized as a simple static support in the treatment of facial paralysis, in digital pullay reconstruction, lip augmentation, and in various nerve palsies as tendon transfer. ${ }^{7,13}$

Anatomical variations of the flexor carpi ulnaris that have been reported previously are: extratendinous bands or extensions from the tendon of the flexor carpi ulnaris to the fourth or more commonly the fifth metacarpal bone; duplication of the flexor carpi ulnaris tendon; a fibrous or muscular extension from the tendon of the flexor carpi ulnaris to the carpal ligaments; the flexor carpi ulnaris diving origin to an accessory abductor digiti minimi muscle. ${ }^{2,21,23}$ However, the flexor carpi ulnaris variation described here is unknown in the anatomical and surgical literature. Clinically, the reported variations of the flexor carpi ulnaris could cause ulnar nerve compression. ${ }^{2,10}$ Moreover, the flexor carpi ulnaris combined with selective release of the flexor pronator origin in patients with hemiplegic cerebral palsy for a pronation flexion deformity of the forearm, hand and wrist. ${ }^{9}$

The median artery is a transitory vessel that represents the arterial axis of the forearm during early embryonic life and normally regresses in the second embryonic month. ${ }^{5,6}$ Its persistence in the human adult has been recorded in two different patterns: as a small and short vessel - antebrachial type (76\%), which ends before reaching the wrist joint; or as a large, long vessel - palmar type (1.5-12\%) which reaches the hand. ${ }^{5,8,24}$ The palmar type is divided into two subtypes: radio-medio-ulnar and medio-ulnar, as in our case. In Bulgarian population the medio-ulnar subtype vary between $5.5-11.1 \% .^{25}$ Rarely, a persistent median artery may occur in conjunction with anomalies in the median nerve such as its high division. ${ }^{6}$ The clinical importance of the persistence of this artery at the wrist level is well documented as a cause of the carpal tunnel syndrome, but it has also been associated with the "pronator teres syndrome" in cases where the persistent median artery pierces the median nerve in the proximal third of the forearm. ${ }^{4,8}$ Other authors speculated that this artery may also play a role in "anterior interosseus nerve syndrome" in cases that immediately after its origin, was intimately related with the nerve. Due to the fact that this artery is responsible for the arterial supply to the median nerve and the neighbouring muscles and as in our case for the thumb and radial side of the index finger its damage could have serious affects to blood circulation of the forearm and hand. ${ }^{6}$

\section{Conclusion}

The aberrant muscles and arteries, including a rare variation of the palmaris longus, undescribed flexor carpi ulnaris variation and a persistent median artery reported in this study may cause entrapment neuropathies, cosmetic defects and functional disability and may have an important role in plastic and reconstructive surgery. Moreover, their combination as in our case, may lead to a complicate clinical appearance and must always be remembered by the clinicians.

\section{References}

1. Wadhwa S, Vasudeva N, Kaul JM. A rare constellation of multiple upper limb anomalies. Folia Morphol (Warsz) 2008; 67: 236-9.

2. Al-Qattan MM, Duerksen F. A variant of flexor carpi ulnaris causing ulnar nerve compression. F Anat 1992; 180: 189-90.

3. Coskun N, Sarikcioglu L, Donmez BO, Sindel M. Arterial, neural and muscular variations in the upper limb. Folia Morphol (Warsz) 2005; 64: 347-52. 
4. Gainor BJ, Jeffries JT. Pronator syndrome associated with a persistent median artery. A case report. 7 Bone foint Surg Am 1987; 69: 303-4.

5. Rodríguez-Niedenführ $M$, Sañudo JR, Vázquez T, Nearn L, Logan B, Parkin I. Median artery revisited. F Anat 1999; 195: 5763.

6. Sañudo JR, Chikwe J, Evans SE. Anomalous median nerve associated with persistent median artery. F Anat 1994; 185: 447-51.

7. Zeybek A, Gürünlüoglu R, Cavdar S, Bayramiçli M. A clinical reminder: a palmaris longus muscle variation. Ann Plast Surg 1998; 41: 224-5.

8. Chalmers J. Unusual causes of peripheral nerve compression. Hand 1978; 10: 168-75.

9. El-Said NS. Selective release of the flexor origin with transfer of flexor carpi ulnaris in cerebral palsy. 7 Bone foint Surg Br 2001; 83: 259-62.

10. Harrelson JM, Newman M. Hypertrophy of the flexor carpi ulnaris as a cause of ulnar-nerve compression in the distal part of the forearm. Case report. F Bone foint Surg Am 1975; 57: 554-5.

11. Kaufmann RA, Pacek CA. Pulley reconstruction using palmaris longus autograft after repeat trigger release. 7 Hand Surg Br 2006; 31: $285-7$.

12. Lin CH, Wei FC, Lin YT, Chen CT. Composite palmaris longusvenous flap for simultaneous reconstruction of extensor tendon and dorsal surface defects of the hand-long-term functional result. f Trauma 2004; 56: 1118-22.

13. Sebastin SJ, Lim AY, Bee WH, Wong TC, Methil BV. Does the absence of the palmaris longus affect grip and pinch strength? $f$ Hand Surg Br 2005; 30: 406-8.

14. Seyhan T. Median nerve compression at the wrist caused by reversed 3-headed palmaris longus muscle: case report and review of the literature. Am 7 Orthop 2005; 34: 544-6.
15. Lisanti M, Rosati M, Maltinti M. Ulnar nerve entrapment in Guyon's tunnel by an anomalous palmaris longus muscle with a persisting median artery. Acta Orthop Belg 2001; 67: 399-402.

16. Sahinoglu K, Cassell MD, Miyauchi R, Bergman RA. Musculus comitans nervi mediani (M. palmaris profundus). Ann Anat 1994; 176: 229-32.

17. Acikel C, Ulkur E, Karagoz H, Celikoz B. Effort-related compression of median and ulnar nerves as a result of reversed three-headed and hypertrophied palmaris longus muscle with extension of Guyon's canal. Scand F Plast Reconstr Surg Hand Surg 2007; 41: 457.

18. Turner MS, Caird DM. Anomalous muscles and ulnar nerve compression at the wrist. Hand 1977; 9: 140-2.

19. Georgiev GP, Jelev L, Surchev L. Reversed palmaris longus muscle simulating soft-tissue mass as a possible cause of median nerve compression. 7 Orthop Trauma (Bulgaria) 2008; 45: 92-4.

20. Georgiev GP, Jelev L, Surchev L. Presence of a palmaris longus related variations in three members of a family. 7 Hand Surg Eur Vol 2009; 34: 277-8.

21. Testut L. Traité d'anatomie humaine. Tome I. Ostéologie Arthrologie - Myologie., Paris: Gaston Doin \& Cie; 1928.

22. Paraskevas G, Tzaveas A, Natsis K, Kitsoulis P, Spyridakis I. Failure of palmaris longus muscle duplication and its clinical application. Folia Morphol (Warsz) 2008; 67: 150-3.

23. Macalister A. Additional observations on muscular anomalies in human anatomy (third series), with a catalogue of the principal muscular variations hitherto published. Trans Roy Irish Acad 1875; 25: $85-6$

24. Adachi B. Das Arteriensystem der Japaner. Vol 1. Kioto: Verl. der Kaiserlich-Japan Univ.; 1928.

25. Kaneff A. Zur Frage der Involution der A. mediana beim Menschen. NTVMI 1958; 3: 521-32.

Correspondence to: Georgi P. Georgiev, MD Department of Anatomy, Histology and Embryology Medical University of Sofia, blvd. Sv. Georgi Sofiiski 1 BG-1431 Sofia, Bulgaria Phone: +359-2-91-72-636; Fax: +359-2-851-87-83 e-mail: georgievgp@yahoo.com

Conflict of interest statement: No conflicts declared. 